\title{
The opto-mechanical performance prediction of thin mirror segments for E-ELT.
}

\author{
Jan Nijenhuis, Roger Hamelinck, Ben Braam, TNO Technical Science, Stieltjesweg 1, 2623 CK \\ Delft, The Netherlands.
}

\begin{abstract}
The mirror segments for the E-ELT and TLT are nearly equal in size and shape (hexagonal, $1.2 \mathrm{~m}$ over flat sides). They are very thin (about $50 \mathrm{~mm}$ ) compared to their size. Supporting these mirrors and obtaining high optical performance is a challenge from design and manufacturing point of view. TNO has designed and build (together with VDL-ETG) three identical prototypes for supporting the mirror segments of the E-ELT. These mirror segments vary in size. Hence the gravity induced deformation of the mirror segments will vary from mirror to mirror segment when no measures are taken. The paper will concentrate on the design and analysis of the design features within the support structure to minimize the mirror deformation due to gravity. These features concern passive and active means to influence the mirror segment shape and to compensate for deformation differences.
\end{abstract}

Keywords: Opto-mechanics, Warping harness, adaptive optics, ELT, M1 support structure.

\section{INTRODUCTION}

The E-ELT is going to be the biggest ground based optical telescope in the world in the coming decades. It will have a primary mirror of $39 \mathrm{~m}$ (hexagonal shape) which consists of 798 hexagonal shaped segments (figure 1) with a nominal width of $1.2 \mathrm{~m}$. The mirror will get a parabolic shape which causes that the individual mirror plan shape will slightly deviate from the perfect hexagon.

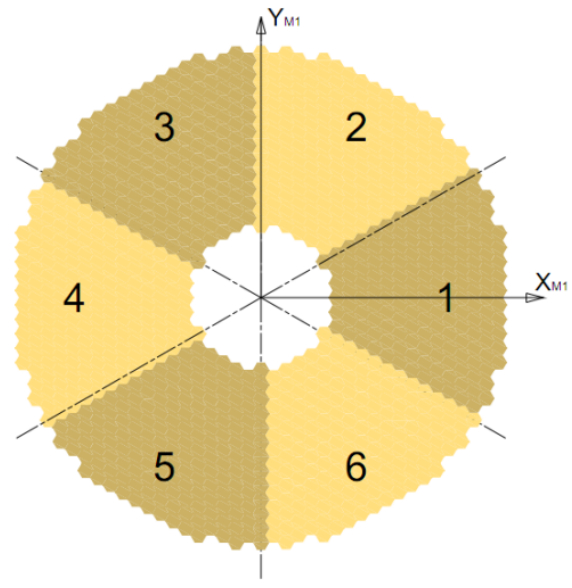

figure 1: Segmentation of mirror M1.
The support structure for each segment will be identical for economic reasons. Inevitably this will result in mirror deformations due to gravity that will be different for each segment. Therefore compensation is needed to minimize the deformation differences. In principle two options exist being active and passive compensation. Both have their (dis)advantages and both will be applied. The clear benefit of passive compensation is that it does not need for electronics, sensors, control etc.

Other sources of static surface form deformations are e.g. manufacturing errors and tolerances of the support structure or the manufacturing errors that are made during manufacturing of the mirror segment. Although the mirror segments will be polished in assembly, still some error will remain that can be compensated for. All these kind of surface form deformation can be compensated by static means. However because they are not known by design they have to be compensated by so called warping harnesses which is a form of active surface control.

Furthermore error sources exist which are related to temperature or the wind speed. These have a dynamic nature and are therefore not predictable and have to be compensated after measurement by active means.

\section{DESIGN DESCRIPTION SEGMENT SUPPORT STRUCTURE.}

A short description of the segment support structure is given below. Only those elements of this structure are described that are needed to understand this paper. Additional information can be found in [1] and [2].

Modern Technologies in Space- and Ground-based Telescopes and Instrumentation II, edited by Ramón Navarro, Colin R. Cunningham, Eric Prieto, Proc. of SPIE Vol. 8450, 84500A (C) 2012 SPIE · CCC code: 0277-786X/12/\$18 · doi: 10.1117/12.926263 


\subsection{The axial mirror segment support}

Each mirror segment is axially supported at 27 support points. These points are grouped into 9 tripods with three support points each. Three tripods are again supported by the Top Level Tripod. Together they form a whiffletree. This is illustrated in figure 2. Such support structure is statically determined which means that the support reaction loads depend only on the whiffletree geometry. The same statement applies to the loads of the segment support struts and the struts connecting the various tripods. The stiffness of the tripods do not influence these loads as long as their deformations are negligible compared to their geometry. The three upper tripods of each whiffletree carry loads of about one third of the lower tripod. Therefore they are also smaller causing that they have much lower mass as the lower tripod.

The 27 support points have been selected by ESO such that the gravity induced deformation has been minimized for the average segment shape. The three support points provide three parallel reaction loads normal to the segment surface thereby constraining three Degrees Of Freedom (DOF) of the mirror segment. These are piston and tip/tilt.

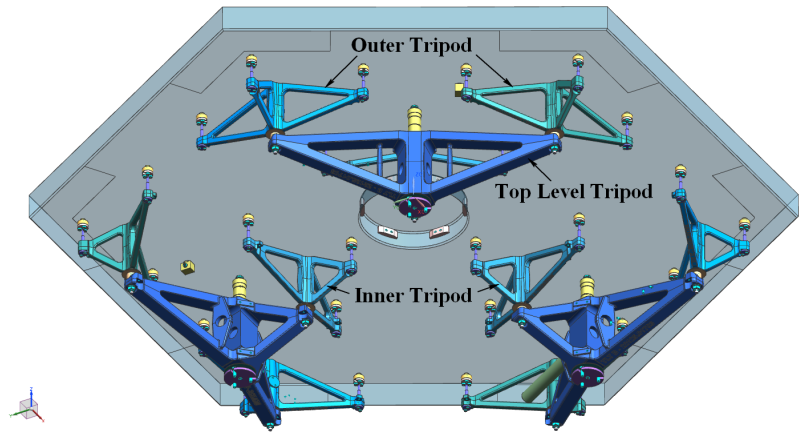

figure 2: The whiffletree support structure of a mirror segment

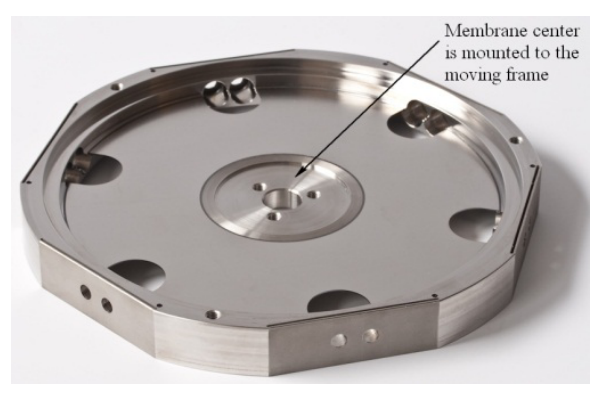

figure 4: Segment membrane.

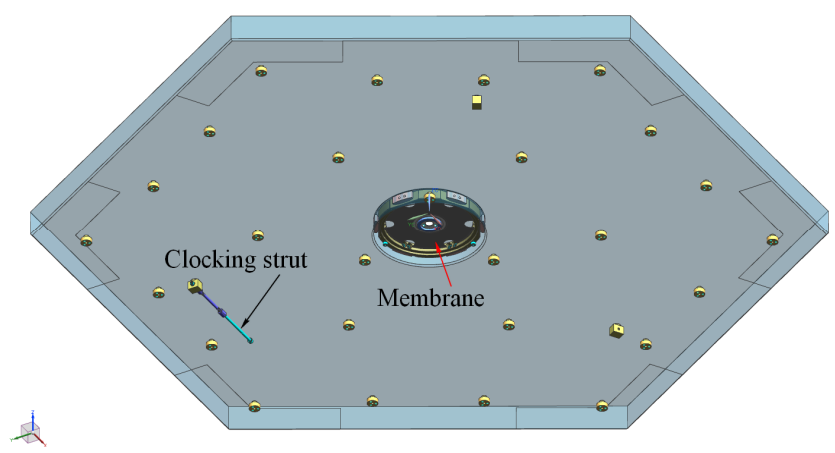

figure 3: Lateral support of the a mirror segment.

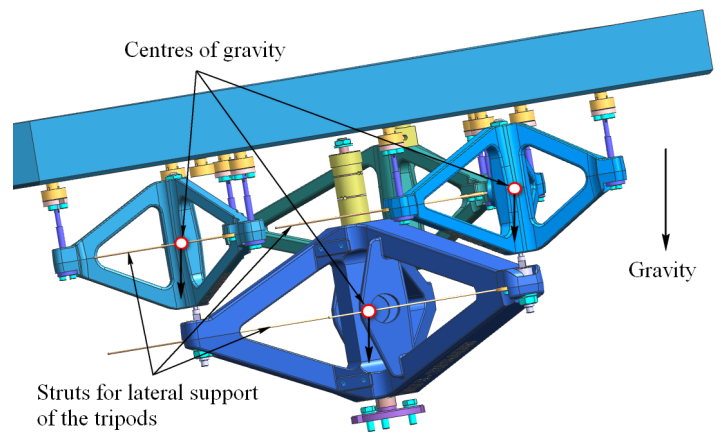

figure 5: Lateral support for whiffletree tripods.

\subsection{The lateral mirror segment support}

To control the other three DOF of the mirror segment as well (translation in X-and Y-direction together with rotation around the Z-axis) a membrane is installed in a pocket in the center of the segment (figure 4). The membrane center is fixed to the moving frame that acts as "fixed world". To enhance its torsion load capability a clocking strut is added at the edge of the segment (figure 3). The other end is connected to the intermediate solid body called moving frame which itself is fixed to "ground". Further details can e.g. be found in [1].

\subsection{Lateral support for the whiffletree tripods.}

Tilting the telescope will cause that the gravity induced loads of the tripods can be decomposed into components parallel and normal to the segment surface (figure 5). By providing lateral struts for the tripods this lateral load is transferred to the moving frame. The figure also illustrates that the center of gravity of each tripod should coincide with the plane that is defined by the lateral support struts. This way no gravity induced bending moments to the segment are created. 


\section{GRAVITY INDUCED MIRROR DEFORMATIONS AND COMPENSATION}

A common way to describe surface form deviation is by use of Zernike coefficients. This allows decomposing the surface from deformation into individual deformation shapes that can be linked to the mechanical support structure. Hence it can be used to optimize the mechanical structure [2]. Piston and tip/tilt are the first Zernike modes and usually the biggest. These are not important for the mirror deformation because they concern rigid body modes that are compensated using the three actuators that support each mirror segment. Higher order deformations like focus, astigmatism, coma etc. are important because these represent actual mirror segment deformations. These can be compensated for by applying moments to the tripods. This will cause out of plane deformations of the mirror segment. There are two option to apply the moments i.e. either by applying a direct torque or by applying a force at certain distance from the tripod.

The way to calculate the best possible surface form compensation is by calculating the deformation caused by individual unit moments applied to the tripod. Because each whiffletree has four tripods this results in eight influence function per whiffletree (figure 6). Hence a total of $3 \times 8=24$ influence functions result. In figure 7 the influence functions are presented for one of the whiffletrees. The influence functions for the other two whiffletrees are identical but $120^{\circ}$ and $240^{\circ}$ rotated. From figure 7 it becomes clear that moments 2 and 20 cause a deformation pattern that is dominated by focus. For moments 1, 7, 9 and 20 this is astigmatism. For each subset of the 24 moments the best fit of the actual deformation is obtained with a linear combination of the influence functions. A set of moments results for each subset and always a small residual error remains.
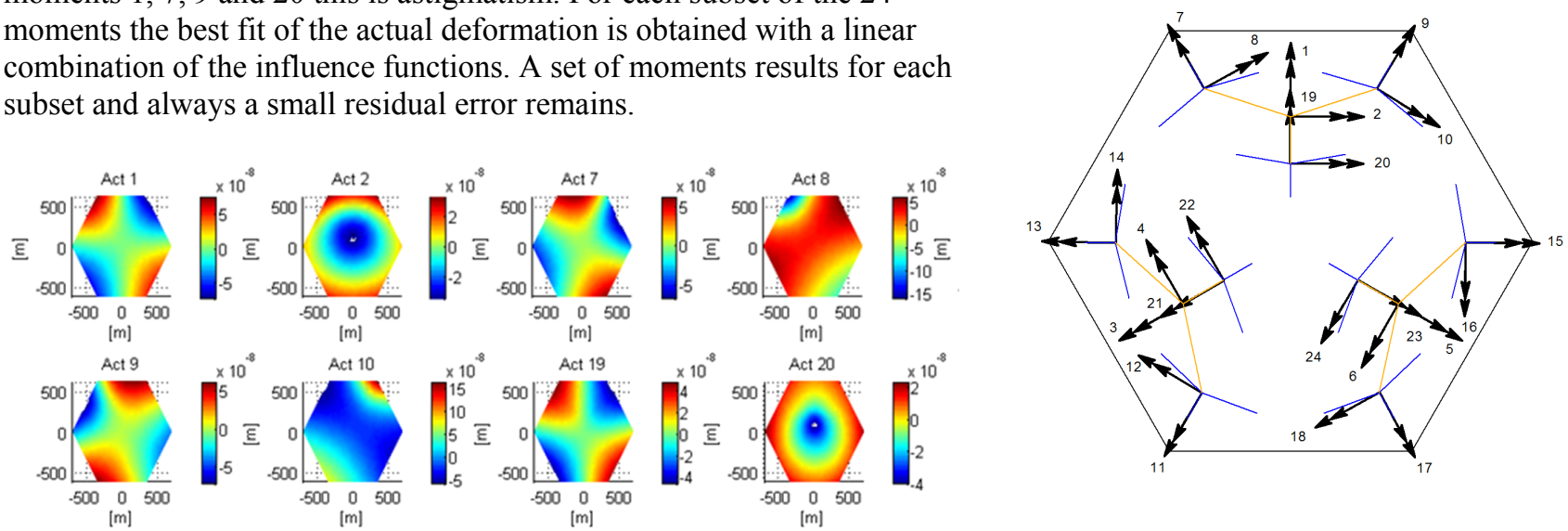

figure 7: The influence function of one of the three whiffletrees.

figure 6: Moments applied to the tripods.

One option to correct for focus error has not been mentioned yet. It was found that a mass mounted at the center of the segment is very efficient in compensation for focus error i.e. with less mass the same focus compensating effect can be achieved.

\section{MINIMIZING GRAVITY INDUCED DEFORMATION WITH PASSIVE MEANS.}

Segment deformation will be dominated by gravity because of the small thickness of the segment $(50 \mathrm{~mm})$ compared to its hexagonal shape of $1.4 \mathrm{~m}$ (corner to corner).. It was required by ESO that this segment deformation should be limited to $30 \mathrm{~nm}$ rms. Only passive means were allowed to realize this. The reason for this requirement is that the telescope will have good optical performance without having to use the active compensation system. During the commissioning phase of the telescope this may prove to be a valuable feature.

The segment deformation decreases when the telescope rotates from zenith to the horizon. Calculating the mirror segment deformation due to gravity and analyzing the results reveals that the segment deformation is dominated by focus and astigmatism. This can be understood when one realizes that the corners of the hexagon have the tendency to hang down (center goes up) when the mirror is bigger than the average (inner mirror segments). The opposite happens for the 
outer mirrors segments because these are relatively small. Clearly this can be recognized as a focus error. Astigmatism occurs because radial and tangential oriented bending of the mirror segment is in general not equal.

From figure 7 it can be concluded that focus is best compensated by applying moments at input locations 2,4 and 6 (figure 6). Moments 20,22 and 24 can do the same but are less efficient because moments 2, 4 and 6 act on all four small tripods of the whiffletree while moments 20,22 and 24 are only applied to one small tripod. Note that the indicated moments not only compensate for mirror segment focus error but also cause higher order deformations. Fortunately the higher order deformations are much smaller in magnitude than the focus error and therefore the total wavefront error of the mirror segment is still reduced.

Astigmatism can be compensated for by applying moment 1,3 and 5 acc. figure 6 . Also moments 7, 9, 11, 13, 15, 17, 19, 21 and 23 can do this but less efficient. Again this is caused by the fact that these moments act on one small tripod only.

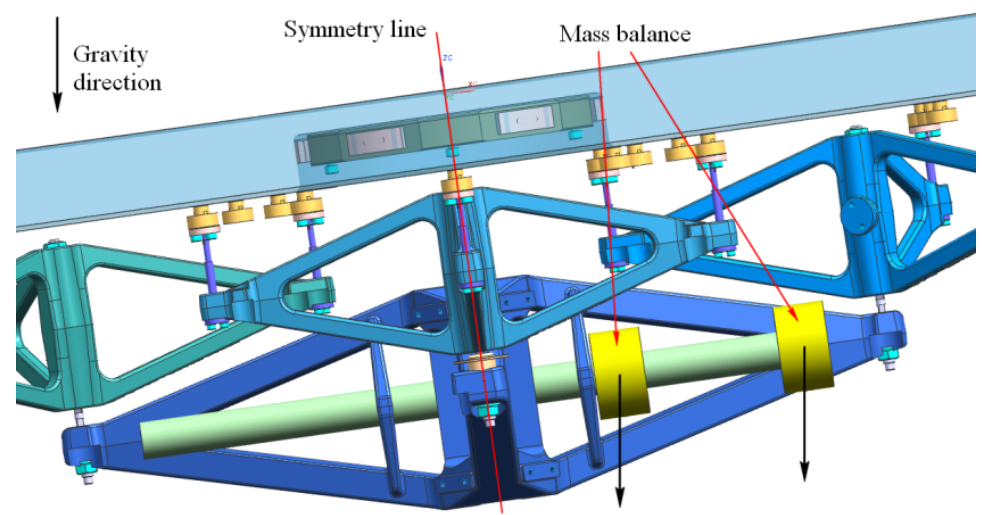

figure 8: Application of mass balance to compensate for astigmatism.
Using balance masses will cause moments to the tripods. Furthermore this moment will be dependent on the gravity direction. This is illustrated in figure 8 . By putting the mass balance either at the LH- or RH-side of the symmetry line any moment up to 2.M.L can be created $(\mathrm{M}=$ mass, $\mathrm{L}=$ moment arm). The gravity vectors of the mass balance can be decomposed into components normal and parallel to the segment surface. Only the normal component causes a segment bending moment. This way the reduction of the balance moment due to mirror segment tilting is equal to the reduction in gravity induced deformation of the mirror. Alternatively springs can be used to create the required balance moments. However this is not angle dependent which is why mass balancing is preferred.

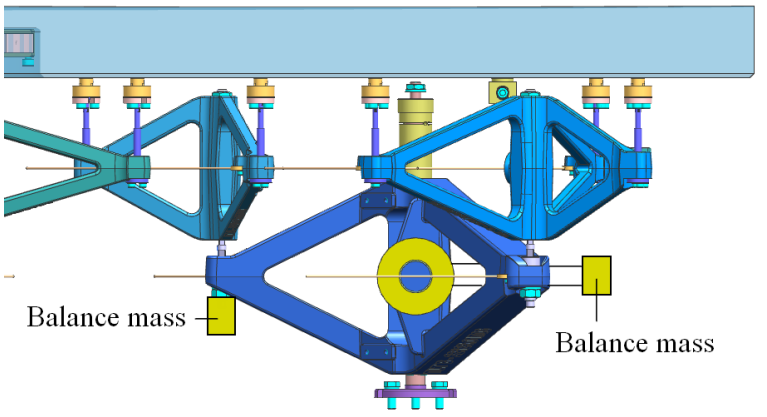

figure 9: Mass balance to compensate for focus.

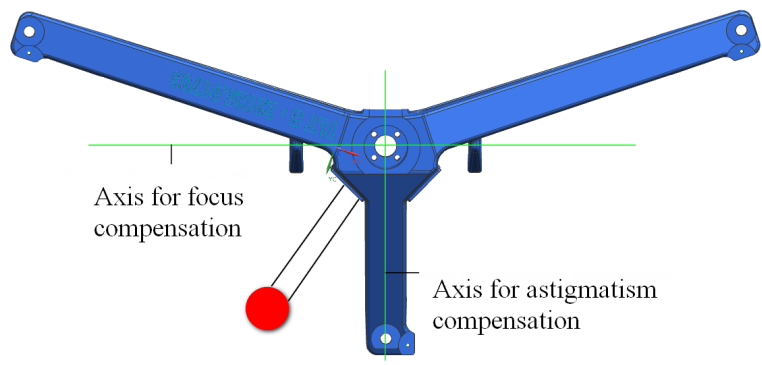

figure 10: Combination of focus and astigmatism correction.

Focus compensation is obtained by mounting the balance masses in the radial direction to the tripod thereby creating bending moments parallel to vector 2,4 and 6 . Focus compensation is shown in figure 9 . From the figure it can be concluded that it is more difficult to create the required moment because the required location of the balance mass is sometimes obstructed by the tripod itself. The alternative is to mount variable balance masses at a fixed position. From an economical and logistical point of view this is not attractive although the total mass that has to be added is minimized provided the moment arm is as long as possible. 
Focus and astigmatism compensation can also be combined (figure 10). This will minimize the total mass for balancing. However it is not always possible to combine the two because the balance mass would be needed where one of the arms of the tripod is located. This option has therefore not been selected.

Another source for mass balancing is the tripod itself. Its COG (center of gravity) is located in-plane with its lateral support (figure 5). By giving it an offset in the radial direction it is possible to create a bending moment that helps minimizing the required additional balance mass for focus correction. Furthermore it proved to be possible to locate the balance mass always to the same side of the tripod ( $\mathrm{LH}$-side in figure 9). As mentioned before it proved to be more mass efficient to use a centrally mounted balance mass instead of separate balance masses attached to the three tripods.

Shifting the COG of the tripods in the tangential direction is not possible because it breaks the symmetry in the design. For each segment there is a mirror imaged one meaning that also the support structure should be mirror imaged which is not allowed. The idea is to build one identical support structure for all mirror segments.

\section{Surface form deformation after correction for focus and astigmatism.}

For three segments the remaining surface form error after passive compensation has been calculated. These segments are located at the inner edge of the M1-mirror, the middle ring and the outer edge. The results clearly demonstrate that the passive compensation can be very effective and that the requirement of $30 \mathrm{~nm}$ rms can be met.
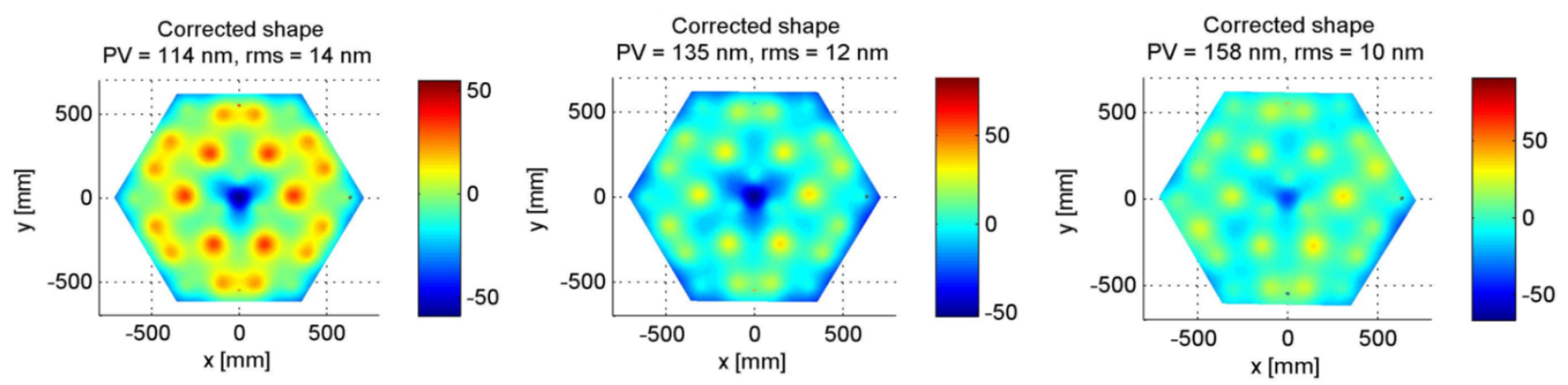

figure 11: Surface form errors after correction.

\section{ACTIVE COMPENSATION FOR GRAVITY DEFORMATION}

Not all surface form errors can be compensated by passive means because they are not predictable. In the introduction already several causes were mentioned. E.g. manufacturing errors due to polishing and manufacturing tolerances of the structure. The latter will cause elastic deformation of the structure which in turn causes small bending moment that will disturb the ideal surface form. These error sources have to be compensated by active means. This requires that the actual surface form of the M1 mirror has to be measured at regular intervals. Based on that result it has to be calculated which moments have to be applied to the tripods. ESO specified that simultaneously $600 \mathrm{~nm}$ PTV focus, $1200 \mathrm{~nm}$ PTV Astigmatism and 300nm PTV trefoil can be reduced by factors 7, 18 and 6 respectively. It has been evaluated for many subsets of the 24 possible moments which one could realize the required performance improvement for the minimal number of compensating bending moment. These moment will be applied using so called warping harnesses and it was found that warping harnesses are needed at position 1-6, 9,13 and 17 (figure 6). Alternatively bending moment to the outer tripods 7, 11 and 15 can also be applied instead because of the symmetry in the mirror segment. The predicted surface form improvement is given in table 1. From the table it can be concluded that a small amount of coma can be compensated too by use of the warping harnesses. 
table 1: Predicted surface form improvements by using warping harnesses.

\begin{tabular}{|c|c|c|c|}
\hline ID & $\begin{array}{c}\text { Description } \\
\text { Zernike mode }\end{array}$ & $\begin{array}{c}\text { WH 1-6, 7, } \\
\mathbf{1 1} \text { and 15 }\end{array}$ & $\begin{array}{c}\text { WH 1-6, 9, } \\
\mathbf{1 3} \text { and 17 }\end{array}$ \\
\hline Mode 4 & Focus & 7,3 & 7,3 \\
\hline Mode 5 & Astigmatism & 20 & 20 \\
\hline Mode 6 & Astigmatism & 20,1 & 20,1 \\
\hline Mode 7 & Coma & 2,7 & 2,7 \\
\hline Mode 8 & Coma & 2,7 & 2,7 \\
\hline Mode 9 & Trefoil & 6,6 & 6,7 \\
\hline Mode 10 & Trefoil & 10,9 & 10,9 \\
\hline
\end{tabular}

The moments that have to be applied to realize the required surface form improvement are given in table 2 . These moments appears to be rather well balanced.

table 2: Required moments to compensate for focus, astigmatism $1 / 2$ and trefoil $1 / 2$ simultaneously.

\begin{tabular}{|c|c|c|c|c|c|c|}
\hline $\begin{array}{c}\text { Actuator } \\
\text { number }\end{array}$ & Focus & $\begin{array}{c}\text { Astigm. } \\
1\end{array}$ & $\begin{array}{c}\text { Astigm. } \\
2\end{array}$ & Trefoil 1 & Trefoil 2 & \\
\hline & 600 & 1200 & 1200 & 300 & 300 & {$[\mathrm{~nm}]$} \\
\hline & & & & & & \\
\hline 1 & $-0,1$ & $-5,2$ & 0,1 & 0,1 & -4 & {$[\mathrm{Nm}]$} \\
\hline 2 & 3,8 & $-0,1$ & $-1,3$ & 0 & 0 & {$[\mathrm{Nm}]$} \\
\hline 3 & 0 & 2,4 & $-4,5$ & 0 & -4 & {$[\mathrm{Nm}]$} \\
\hline 4 & 3,6 & 1,2 & 0,6 & 0,1 & 0 & {$[\mathrm{Nm}]$} \\
\hline 5 & 0,1 & 2,7 & 4,3 & 0,1 & -4 & {$[\mathrm{Nm}]$} \\
\hline 6 & 3,9 & $-1,1$ & 0,8 & 0,1 & 0 & {$[\mathrm{Nm}]$} \\
\hline 9 & 0 & 0,3 & 0,6 & $-3,4$ & 0 & {$[\mathrm{Nm}]$} \\
\hline 13 & 0,1 & $-0,7$ & 0 & $-3,5$ & 0 & {$[\mathrm{Nm}]$} \\
\hline 17 & 0,2 & 0,3 & $-0,5$ & $-3,3$ & 0 & {$[\mathrm{Nm}]$} \\
\hline
\end{tabular}

Applying the required moments to the tripods can be done in two ways. Either by applying a pure moment or by applying a force at certain distance. Both options are illustrated in figure 12. Both will cause that the moving frame and whiffletrees will be loaded internally i.e. the loading of the three support actuators will not be influenced. Which of the two options is implemented depends on the design options to implement it. TNO has selected to apply torsion moments to the tripods by use of a clocking spring. A stepper motor with self-locking gearbox and rotating output shaft winds up the spring either in clockwise or counter clockwise direction (figure 14 and figure 16). 


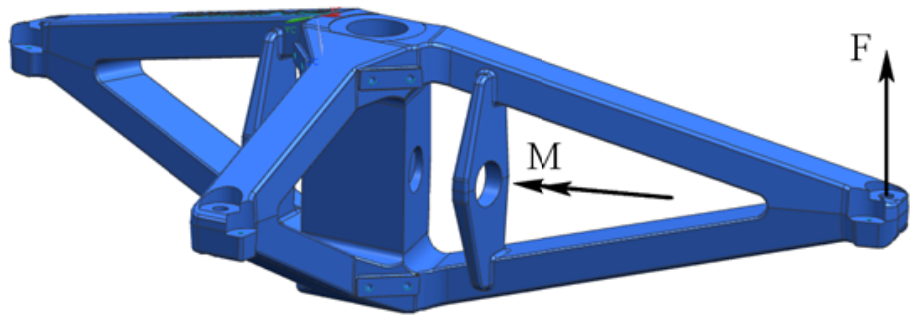

figure 12: Applying a load or a torsion moment to the tripod.
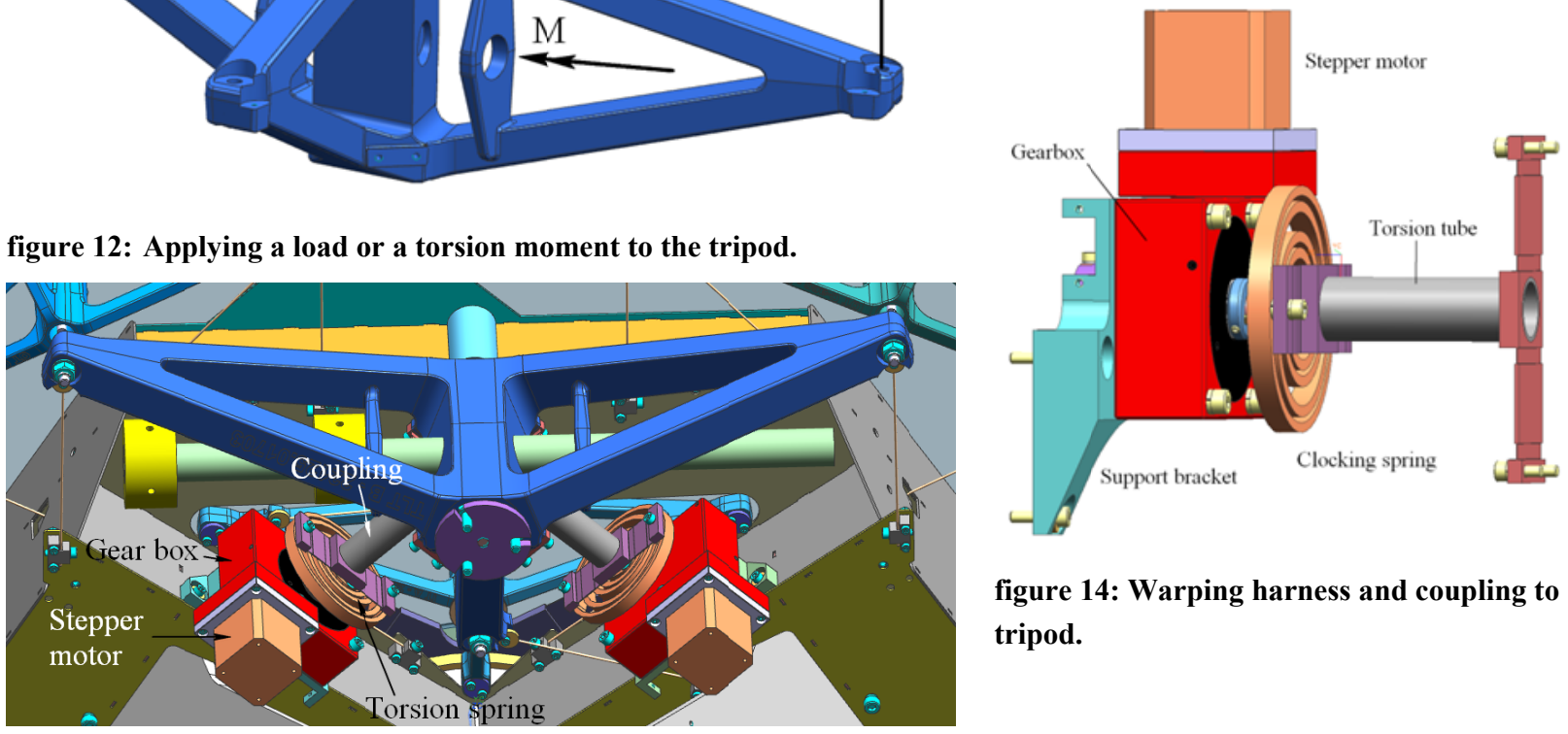

figure 14: Warping harness and coupling to tripod.

figure 13: Warping harnesses mounted to the tripods.

The position of the output shaft is measured using a potentiometer. It measures the absolute position and is not sensitive to power failurest. The coupling to the tripod is done using a set of flexures to guarantee that mechanical tolerances do not cause that spurious moments or forces are exported to the tripod.

E.g. the distance between the moving frame and tripod can easily vary by few tenth of mm thereby creating a gap or lack of space to mount the warping harness. Forcing the warping harness in place would cause a high lateral load to the tripod because of the stiff lateral tripod support. This is to be avoided. With present design of the WH-coupling it is guaranteed that mechanical tolerances cannot cause surface form deviations more than $1 \mathrm{~nm}$ rms.

The warping harness of the outer tripods looks a little different because of space limitations (figure 15). From a functional point of view nothing has changed. Especially the flexible coupling has changed.

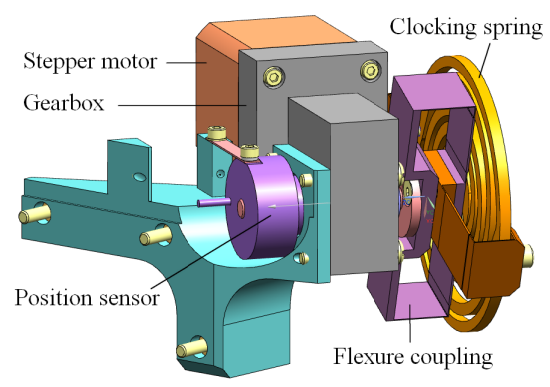

figure 15: Warping harness for the outer tripods.

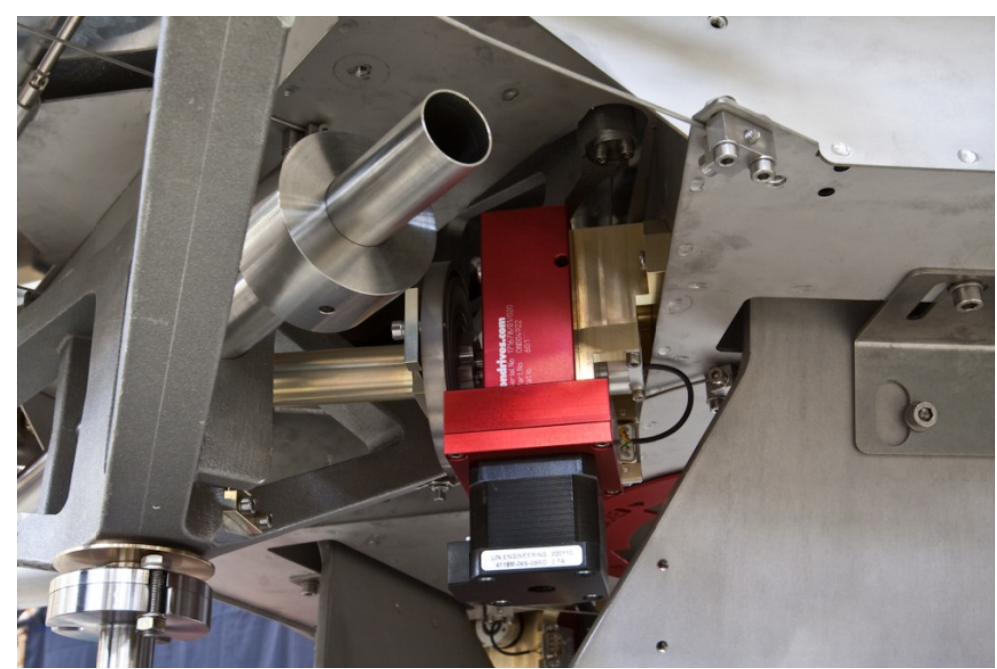

figure 16: Warping harness as build into the system. 


\section{THERMAL INDUCED MIRROR SEGMENT DEFORMATIONS.}

The thermal expansion difference between segment and support structure will cause that 27 tangential oriented bending moments are introduced at the strut to segment connection which causes a focus deformation of the mirror segment. Because of the difference in thermal expansion between the mirror segment (near zero) and the metal support structure it becomes difficult to compensate for thermal induced deformations in the radial direction. Therefore it is better to make the design insensitive to it. This is obtained by making the 27 support struts of the segment rather long compared to their diameter. This way maximum use is made of the high tensile stiffness of such strut compared to its bending stiffness. However also the absolute value for the bending stiffness should be low. Therefore this effect should be minimized by making the ratio of bending to tension stiffness as small as possible. The bending and tension stiffness of the strut are given by:

$$
\text { Bending stiffness } \quad \frac{12 \cdot E \cdot I}{l^{3}}=\frac{3 \cdot \pi \cdot E \cdot d^{4}}{16 \cdot l^{3}} \quad \text { Tension stiffness } \quad \frac{E \cdot A}{l^{2}}=\frac{\pi \cdot E \cdot d^{2}}{4 \cdot l^{2}}
$$

Whereby:

E: $\quad$ Youngs Modulus.

d: $\quad$ Diameter of strut.

A: $\quad$ Area of cross section of strut.

I: $\quad$ Moment of inertia of cross section of strut.

1: $\quad$ Length of strut.

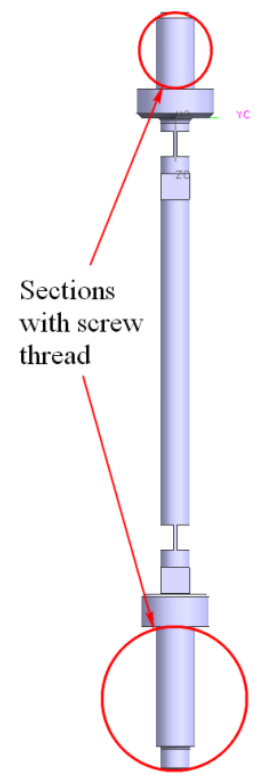

Hence their ratio is therefore proportional to $(\mathrm{d} / \mathrm{l})^{2}$.

Another option is to use a strut with two thin end sections instead of a prismatic strut (figure 17).

figure 17: Strut with thin end sections.

This thin section has width identical to the strut diameter but its thickness is much less. Length of the section is small compared to the total length of the strut. The thickness of center section of the strut is more than that of a comparable prismatic strut. This way the stiffness properties of the strut are fully determined by these end sections. It can be shown that now the bending to tension stiffness of the strut is proportional to $(t / l)^{2}$. Because thickness is much less than the prismatic rod diameter a segment support strut can be realized that e.g. is much shorter for the same lateral stiffness and therefore allows a more compact design.

\section{CONCLUSION}

This paper gives an overview of the support structure for the main E-ELT mirror. The high demands on mirror form accuracy in combination with changing gravity can only be met by a combination of active and passive compensation of the disturbances. Summarizing it has been shown in this article that:

- Compensation of gravity induced deformations by use of passive means is possible.

- The design of the passive compensation system is such that tilting of the mirror segments has an equal effect on compensation and gravity induced deformation.

- Applying bending moments to the central tripod of the whiffletree is very efficient. It limits the number of required warping harnesses.

- Active compensation of surface form deviations can be done by introducing internal moments in the structure between moving frame and tripod.

- Using segment support struts with reduced thickness of the end sections facilitates a compact design of the segment support structure. 


\section{REFERENCES}

[1] J. Nijenhuis and R. Hamelinck, "Meeting highest performance requirements for lowest price and mass for the M1 segment support unit for E-ELT," in SPIE Astronomical instrumentation, San Diego, 2010.

[2] J. Nijenhuis and R. Hamelinck, "The optimization of the opto-mechanical performance of the mirror segments for the E-ELT.," in Integrated modelling conference, Kiruna, 2011. 